\title{
Distribution pattern of the pancreatic amylase around Golgi area, demonstrated by means of immuno-electron microscopic method
}

\author{
by \\ Kenjiro Yasuda*, Toshio Suzuki* and Kunio Takano** \\ * Department of Anatomy, School of Medicine, Keio University, \\ Shinjuku, Tokyo, Japan \\ ** Department of Anatomy, School of Dentistry, Nihon University, \\ Chiyoda, Tokyo, Japan
}

\begin{abstract}
The biochemical investigation has proved the high amylase activity in the zymogen granules of the pancreatic acinar cells. Since these results are based on the measurement of the enzymatic activity in each fraction of the cellular component separated by the ultra-centrifugation, amylase activity was also encountered even in the supernatant as well as in the nuclear fraction ( $\mathrm{Hok} \mathrm{in}, 1955)$. This may not be the direct proof of the presence of amylase in the free cell sap, but may possibly be due to the release of the enzyme from the cell organelles during the course of the preparation of the cell fractions. While, the morphological evidence demonstrated by the fluorescent antibody method ( $\mathrm{Y}$ a s u d a and $\mathrm{C}$ o o n s, 1966) showed the presence of the specific immunofluorescence not only in the zymogen granules but also in the juxta-nuclear cytoplasm. The present study was confined to investigate the localization of amylase in the electron microscopic level, specifically around the Golgi area, by using ferritin-tagged antibody method.
\end{abstract}

\section{Materials and methods}

The material used comprised the pig pancreas obtained from the slaughter house. Small blocks of the pancreatic tissue were further sliced in pieces with razour blade and pre-fixed for one hour in buffered $5 \%$ formalin solution containing $0.001 \mathrm{M}$ EDTA and $0.25 \mathrm{MI}$ sucrose. After the pre-fixation, the tissue was applied to the ferroglobulin solution prepared against porcine pancreatic amylase, according to the method described by $\mathrm{S}$ inger (1959). Then. the small 
blocks were washed three times with Tyrode solution, followed by the post-fixation in the $\mathrm{OsO}_{4}$ solution. Dehydration and embedding in Epoxy resin were carried out as the common technique for the electron microscopy. As the control, heterogenic antibodies, i.e., anti-vaccinea virus serum and anti-chymotrypsin serum, were also tagged with ferritin and used for the staining in the same manner as described above.

\section{Observation and discussion}

The ferritin granules, which represent the site of the antigen indirectly, are encountered in the space surrounded by the lamellar structure in the Golgi area (Fig. 1 and 2), in the vacuoles (Figs. 2, 3 and 4 ) and in the multivesicular bodies (Figs. 5, 6, 7 and 8) around the Golgi area. As seen in Figs. 1 and 2, the homogeneous material enclosed within the lamellar structure is much denser than the surrounding area and is heavily laden with ferritin granules. The dense area is, on one side, always sharply bordered by the Golgi lamellae, but, on the other side, is occasionally continuous with the material which is also opaque and diffusely distributed within or around the Golgi area. It is not obvious whether this diffuse material is on the course of the condensation of amylase into the Golgi area from elsewhere in the cytoplasm or is diffusing out of the Golgi area. It is widely accepted that the proteins, including enzymes, are generally synthesized in or on the surface of the rough-surfaced endoplasmic reticulum, transfered into the Golgi apparatus, and condensed there to be the young secretory granules. But, the course of the transportation of the newly synthesized protein from the rough-surfaced reticulum to the Golgi apparatus is still obscure. There have been several explanations as regard to the mechanism of the transportation of the newly synthesized protein ( $\mathrm{P}$ a l a d e, 1962, K u rosum i, 1962, and $\mathrm{Ku}$ rosum i, 1965). Summarized from the above mentioned papers, one possibility is the budding-off of a part of the rough-surfaced reticulum after loosing the ribosomes on its surface. Then the budding is followed by the pinching-off of the smooth-surfaced projection from the rough-surfaced reticulum, being given rise to the small vesicles which are eventually called Golgi vesicles. Another possibility is the direct communication of the rough-surfaced reticulum with the Golgi apparatus. Apart from the discussion on the mechanism of the transportation, the presence of the massive ferritin granules in the amorphous cytoplasm around 
the Golgi lamellae (Figs. 1 and 2) is often recognized. It is awfully hard to imagine the nature of amylase which is not associated with any kind of the cell organelles. This may be explained in some of the following ways; amylase produced somewhere in the cytoplasm, for example, in the rough-surfaced cisternae, is once removed into the free cytoplasm near the Golgi area to wait to be transfered into the Golgi apparatus; amylase synthesized in the rough-surfaced cisternae near the Golgi area diffused out of the cisternae toward Golgi apparatus, or amylase concentrated in the Golgi area is released from that area during the course of the experiments. As mentioned above, to pick up one possibility out of three is almost impossible. Supposed, there were no artificial disorientation of the cell organellae even after the double fixation with the intervened period of the staining the prefixed tissue with ferroglobulin and following rinsing with Tyrode solution, this figure might suggest the diffusing of amylase from rough-surfaced cisternae toward Golgi apparatus through the amorphous cytoplasm. In other words, temporary presence of amylase in free cell sap could be imagined, as $\mathrm{H}$ o k i n (1955) had suggested.

The heavy aggregation of ferritin granules in the definite area bordered half round by Golgi lamellae represents also the site of amylase (Figs. 1, 2 and 3). Unless the specimens are not stained for amylase, the area mentioned above is difficult to be distinguished from the other part of cytoplasm under the regular electron microscopy. Though the membraneous structure is not clearly seen around the proper area in Fig. 3, this area might be bounded by the smoothsurfaced membrane which is continuous to the Golgi lamellae. Namely, the material which is observed as the dense mass is amylase itself and occupies the interspace between membranes of the Golgi lamellae. In other words, the material or amylase is not coagulated in the cytoplasm in a mass but is surrounded by the membraneous structure, though occasionally difficult to be recognized on the electron micrograph (Fig. 3).

The two facts described above are not enough to give an answer to the question whether a part of amylase is present in the free cytoplasm or not.

Amylase is also found in the multivesicular body (Figs. 4-9), as well as in the vesicles and vacuoles around the Golgi area. The structure and nature of the multivesicular body are firstly described by Sote 10 and Porter (1959). Occasionally, the inner vesicles of the multivesicular bodies do not contain any ferritin granules (Figs. 
6 and 7). It might possibly be very difficult for ferritin to penetrate the membranes of the multivesicular body to go into the inner vesicles. Therefore, the negative reaction of the inner vesicles (Fig. 7 ) is not reliable as far as the enzyme localization is concerned.

The dense structure composed of vacuoles and lamellar arrangement of the smooth membrane in Fig. 10 is supposed te be so-called dense body which might be closely related to the multivesicular body or to the lisosomes. This structure does not contain any amylase in it, though it is reported to have acid phosphatase activity ( $\mathrm{K} \mathrm{u} \mathrm{r} \mathrm{o-}$ $\mathrm{s} u \mathrm{~m} \mathrm{i}, 1965$ ). If the lisosomes were originated from Golgi apparatus and further turned into the dense body, the dense body might possibly have contained amylase, since amylase is distributed in several structures associated with Golgi apparatus. Nevertheless, as mentioned above, the dense body did not contain any amylase in it. Though the staining of acid phosphatase was not carried out in this study, acid phosphatase and amylase may not co-exist in the dense body. Because, acid phosphatase is presumed to be localized in the disintegrating area of a cell or the region where the proteolytic activity is being took place ( $\mathrm{K} \mathrm{ur}$ os u mi, 1965), on the other hand, amylase is, at least in the pancreas, made in the cell and is destined to be secreted out of the cell. Therefore, amylase may not be present in association with the disintegrating organelles together with acid phosphatase.

\section{Conclusion}

The localization of pancreatic amylase in the acinar cells of the porcine pancreas was examined by means of immuno-electron microscopic method. The observation was particularly confined in the structure around the Golgi apparatus.

The diffuse material which was observed in or around the Golgi apparatus was heavily associated with ferritin granules. This feature might be suggestive of the presence of amylase in the free cytoplasm, though the exact location was still open to question because of the several possibilities to cause the dislocation of the enzyme within the cell. Amylase was also encountered in the multivesicular bodies, but was not recognizable in the dense bodies. 


\section{Literature}

Hokin, L.H.: Isolation of zymogen granules of dog pancreas and a study of their properties. Biochim. Biophys. Acta, $18:$ 379-388, 1955.

$\mathrm{Kur}$ osumi, K. and Koba y a shi, Y.: Morphological variations of the cytoplasmic membrane system in the gland cells of the adenohypophysis. Fifth Int. Congr. Micr. TT-11, "Electron Microscopy" vol. 2, 1962, Academic Press, New York.

Kurosumi, K.: Golgi apparatus and its derivatives, with special reference to secretory granules. Intracellular membraneous structure, pp. 259-276, 1965, ed. by S. Seno and E. V. Cowdry, Japanese Society for Cell Biology, Okayama. (in Japanese)

$\mathrm{Kurosumi}, \mathrm{K}$ : The fine structure of the cell and the transportation of the materials within and out of the cell. J. Clin. Science: 1 (3):1-17, 1965. (in Japanese)

$\mathrm{Palade}$, G.E., Siekevit $z$, P. and Caro, L.G.: Structure, chemistry and function of the pancreatic exocrine cell. Ciba Foundation Symposium on the Exocrine Pancreas. J. \& A. Churchill Co., London, pp. 23-55, 1962.

Singer, S. T.: Preparation of an electron-dense antibody conjugate. Nature, 183: 1523-1524, 1959.

Sotelo, J.R. and Porter, K.R.: An electron microscopic study of the rat ovum. J. Biophys. Biochem. Cytol., 5 : 327-342, 1959.

Yasuda, K. and Coons, A.H.: Localization by immunofluorescence of amylase, trypsinogen and chymotrypsinogen in the acinar cells of the pig pancreas. J. Histochem. Cytochem., 14:303-313, 1966.

\section{Explanation of figures}

Fig. 1. The area surrounded by the Golgi lamellae $(G)$ is filled with dense material which is laded with ferritin granules (f). Namely amylase is encapsulated by lamellar structure of the Golgi apparatus. Beside the dense material mentioned above, there are also opaque masses (om) distributed diffusely around the Golgi area. These masses are also attached by the ferritin granules, and are suggestive of the presence of amylase in the free cytoplasm, assuming that the tissue is not disrupted by the several treatments before or after the antigen-antibody reaction had been took place within the tissue. rer: rough-surfaced endoplasmic reticulum. Magnification: 75,000 $\times$.

Fig. 2. The material enclosed within the Golgi lamellae $(G)$ is heavily associated by ferritin granules (f) which represent the site of amylase. The coarse material $(\mathrm{cm})$ which is found in the free cytoplasm in the surrounding of the Golgi apparatus is also tagged with ferritin, indicating that the material consists of amylase. Magnification: 75, $000 \times$.

Fig. 3. This is the view around the Golgi area and is quite similar to that showed in Fig. 1 or 2. But, there is no amylase which locates in the free cytoplasm. The mass covered half round by the Golgi lamellae combines with ferritin. Amylase is also concentrated in the vesicles (v). $z$ : zymogen granules, $\mathrm{m}$ : mitochondria. Magnification: $60,000 \times$.

Figs. 4 and 5. Several features of the concentration of amylase in the vesicles (v). The location of amylase is visualized by the ferritin granules. $z: z y m o g e n$ granules. Magnification : $75,000 \times$.

Figs. 6-9. Amylase is also localized in the multi-vesicular body. The enzyme is 
mostly concentrated in the inner vesicles, and the space between the inner vesicles are free from enzyme. Some of the inner vesicles are devoid of enzyme, while others are rich in enzyme. Magnification : 75,000 $\times$.

Fig. 10. The structure which might fall into the categoly of the dense body (d) does not contain any amylase, while the vesicles (v) are filled with the enzyme. rer: rough-surfaced endoplasmic reticulum, $z: z y m o g e n$ granule. Magnification : $75,000 \times$. 
Plate I

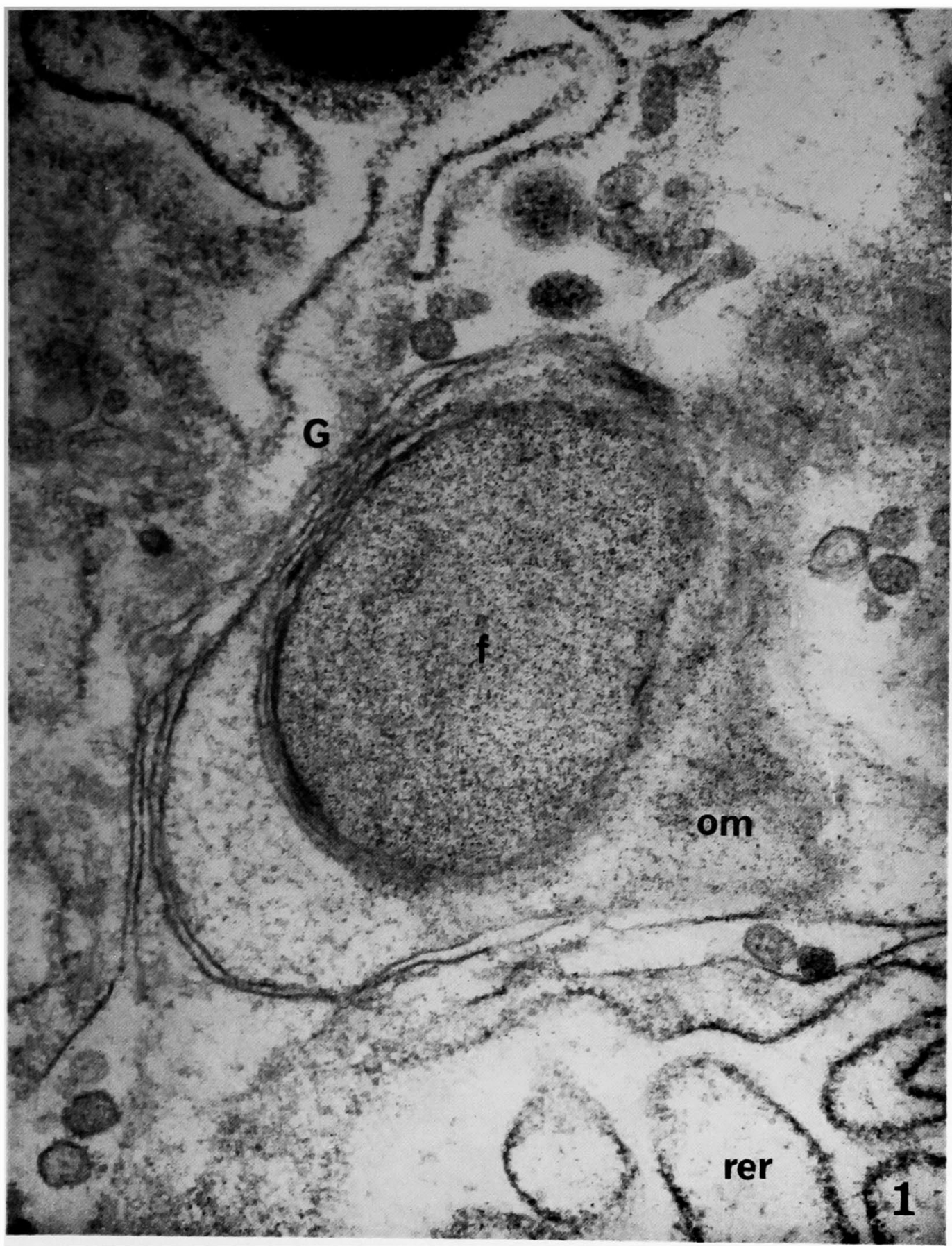

K. Yasuda, T. Suzuki and K. Takano. 
Plate II

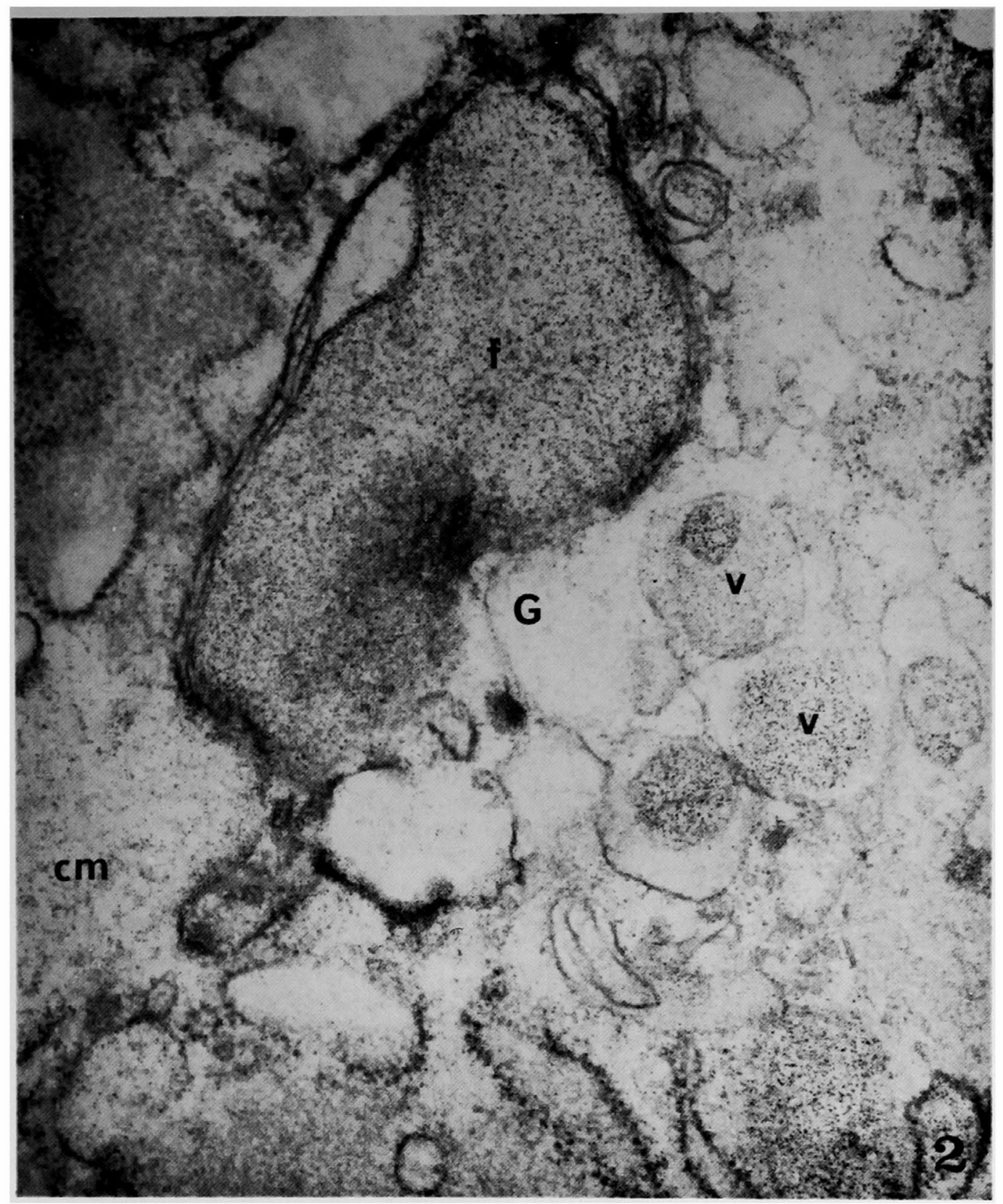

K. Yasuda, T. Suzuki and K. Takano. 


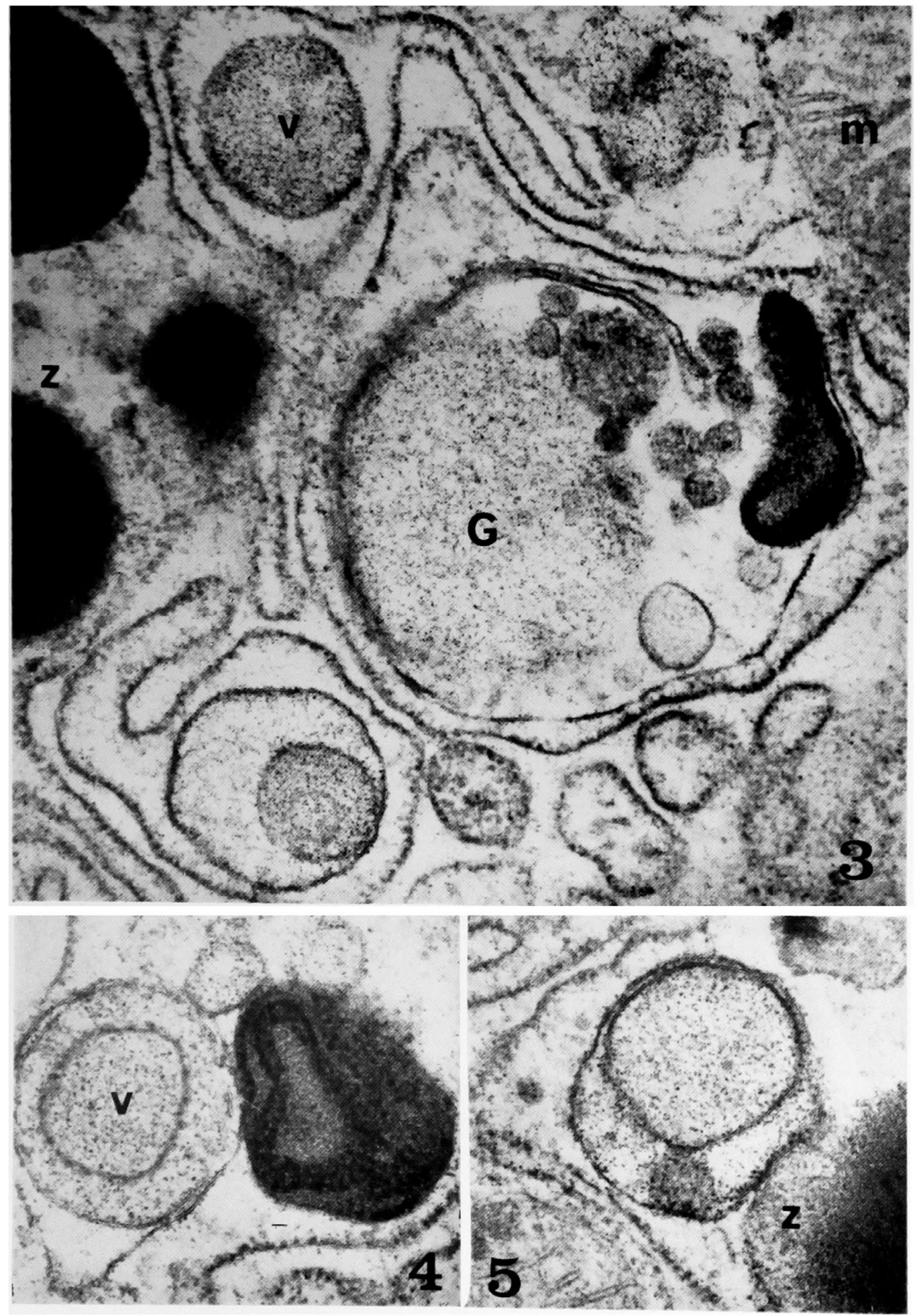

K. Yasuda, T. Suzuki and K. Takano. 
Plate IV
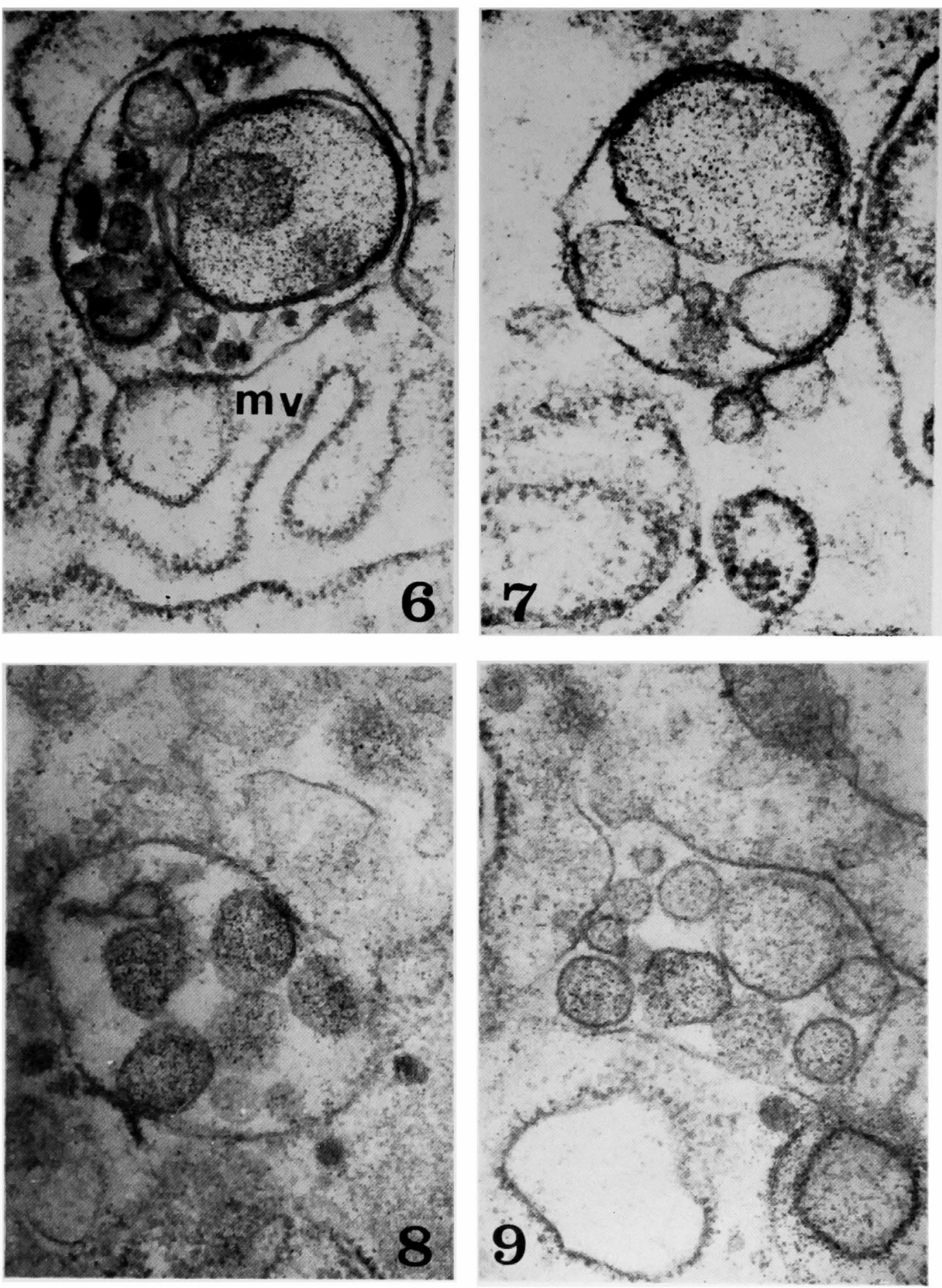

K. Yasuda, T. Suzuki and K. Takano. 


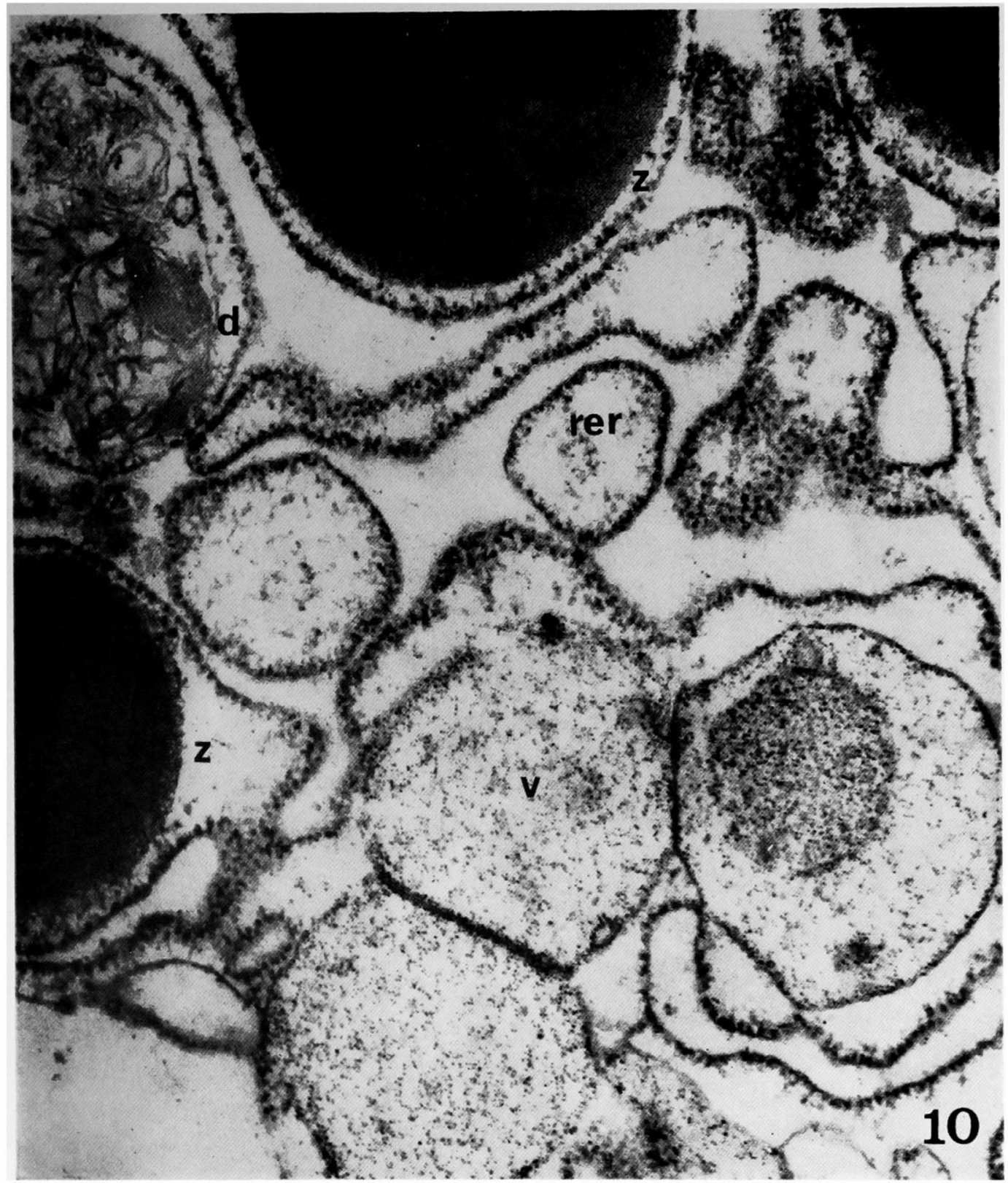

K. Yasuda, T. Suzuki and K. Takano. 